\title{
Los desafíos de la educación médica en la Universidad Veracruzana
}

\section{The challenges of medical education at the Universidad Veracruzana}

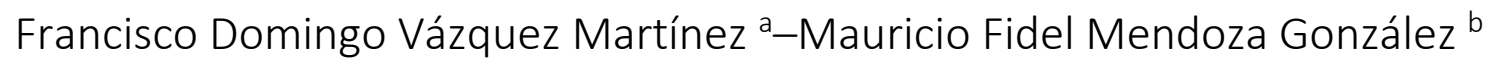

a Responsable del Observatorio de Educación Médica y Derechos Humanos, Instituto de Salud Pública, Universidad Veracruzana, Xalapa, México.

Contacto: obeme@uv.mx

b Instituto de Salud Pública, Universidad Veracruzana, Xalapa, México.

Contacto: mmendoza@uv.mx

Recibido: 14 de agosto de 2020

Aceptado: 15 de octubre de 2020

RESUMEN: El presente trabajo actualiza la principal información generada por el Observatorio de Educación Médica y Derechos Humanos (OBEME): 1) la mayoría de las y los estudiantes de medicina (internos, pasantes y residentes) de la Universidad Veracruzana en las unidades médicas informan no estar siempre supervisados y reconocen haber cometido errores diagnósticos o terapéuticos, precisamente por esta causa; 2) la acreditación de las facultades de Medicina contrasta con el hecho de que solo una de las cinco facultades garantiza con evaluaciones externas la formación profesional de sus egresados; y 3) respecto a los desafíos que plantea la pandemia a la educación médica se resalta la necesidad de recuperar la formación deontológica en medicina, incorporar la gestión de epidemias y desastres sanitarios en el plan de estudios y garantizar la salud física y mental de los estudiantes de medicina durante su formación.

Palabras clave: Educación médica; acreditación programas educativos; COVID-19; errores médicos; seguridad del paciente.

ABSTRACT: This work updates the main information generated by the Observatory of Medical Education and Human Rights (OBEME): 1) Most of the medical students (interns, interns and residents) of the Universidad Veracruzana in the medical units report not being always supervised and acknowledge having made diagnostic or therapeutic errors, precisely for this 
reason; 2) the accreditation of the Faculties of Medicine contrasts with the fact that only one of the five faculties guarantees the professional training of its graduates with external evaluations; and 3) regarding the challenges posed by the pandemic to medical education, the need to recover deontological training in medicine, incorporate the management of epidemics and health disasters into the curriculum, and guarantee the physical and mental health of students is highlighted. of medicine during their training.

Keywords: Medical Education; Accreditation of Educational Programs; COVID-19; Medical Errors; Patient Safety.

\section{Introducción}

1 Observatorio de Educación Médica y Derechos Humanos (OBEME) se fundó en agosto J de 2017. Su objetivo es contribuir a mejorar la calidad, pertinencia y responsabilidad El OBEME es la respuesta del Instituto de Salud Pública de la Universidad Veracruzana (UV) a las múltiples y reiteradas recomendaciones de la Comisión Nacional de Derechos Humanos (CNDH) y de la Comisión Estatal de Derechos Humanos de Veracruz respecto a la falta de supervisión de los estudiantes de medicina (internos, pasantes y residentes) que tienen contacto directo con los pacientes, y a la falta de competencias profesionales de los médicos para diagnosticar e indicar tratamientos adecuados a los pacientes (CNDH, 2009).

Con estos señalamientos en cuenta, es evidente que cualquier esfuerzo por garantizar el derecho a la salud en México, para mejorar el sistema de salud y, en particular, la seguridad de los pacientes y la calidad de la atención médica pasa por garantizar el derecho a la educación en la formación médica y perfeccionar los procesos de educación médica, de manera relevante, aquellos en los que intervienen pacientes.

En el presente trabajo se actualizan los resultados de las investigaciones realizadas por el OBEME respecto a la supervisión de los estudiantes de medicina en contacto directo con los pacientes, y sobre los resultados de los egresados de la carrera de Medicina en las evaluaciones nacionales. También, dada la situación generada por la pandemia de COVID-19 se exponen algunos de los nuevos desafíos que enfrenta la educación médica.

Desde el Enfoque de la Salud Basada en Derechos Humanos (ESBDH) (Yamin A, Frisancho A. 2015) la formación de médicos $y$, en general, de personal de salud es fundamental para garantizar el derecho a la salud. Esto es, sin el pleno goce del derecho a la educación de los 
Observatorios - Observatorio de Educación Médica y Derechos Humanos

estudiantes de medicina no será posible garantizar el derecho a la salud. En educación médica la unicidad de los derechos humanos es evidente.

La interrelación del derecho a la educación y del derecho a la salud se concreta en la supervisión de los estudiantes de medicina que desarrollan procesos formativos con pacientes en las unidades médicas. La supervisión de estudiantes de medicina durante sus actividades diagnósticas y terapéuticas en las unidades médicas es, simultáneamente, parte del derecho a la educación y parte del derecho a la salud.

La falta de supervisión de los estudiantes de medicina en las unidades médicas es una violación al derecho a la educación a la par que transgrede el derecho a la salud; constituye un factor de riesgo para la seguridad de los pacientes, atenta contra la calidad de la atención médica y repercute negativamente en la competencia profesional de los nuevos médicos.

En este sentido, se entiende que sea fundamental para la UV conocer el grado de supervisión de sus internos, pasantes y residentes en las unidades médicas, así como, por otra parte, evaluar el desempeño de sus egresados en las pruebas nacionales disponibles. Lo anterior no porque los señalamientos de la CNDH o de la CEDH-Veracruz se dirijan directamente a ella, sino porque, como garante del derecho a la educación, tiene la obligación porque su compromiso con la responsabilidad social universitaria así lo exige y porque el Consenso Global sobre la Responsabilidad Social de las Facultades de Medicina así lo recomienda (Boelen Ch, Woollard R. 2011).

\section{Supervisión de estudiantes de medicina en las unidades médicas}

De acuerdo con la normatividad vigente los internos, pasantes y residentes, siempre deben estar asesorados y supervisados durante el desarrollo de sus actividades en las unidades médicas. Hay que tener presente que el internado y el servicio social son parte del programa educativo de Licenciatura en Medicina General y que las residencias médicas son programas educativos universitarios para la formación de médicos especialistas. En este sentido, la supervisión de los estudiantes de medicina en las unidades médicas es un proceso conjunto entre las unidades médicas y las instituciones educativas. Las unidades médicas deben supervisar para evitar daños a los pacientes atendidos por estudiantes y las instituciones educativas para responder a la sociedad sobre el cumplimiento de los programas educativos y el logro de las competencias profesionales de todos los egresados de sus programas académicos. 
El OBEME realiza una encuesta desde el ciclo escolar 2017-2018 entre internos, pasantes y residentes de la UV para conocer el cumplimiento de la supervisión de sus estudiantes en las unidades médicas y de los programas académicos en las mismas. Los resultados de la encuesta 2017-2018 están publicados y son de acceso libre (OBEME, 2019). En las tablas 1 y 2 se observa un adelanto de los resultados de la encuesta 2018-2019.

En la tabla 1 se aprecia que, en general, en las unidades médicas se prioriza la atención médica sobre el proceso de enseñanza-aprendizaje y el cumplimiento de los programas académicos. Diez por ciento de internos, $15 \%$ de pasantes y 11\% de residentes consideran que el proceso educativo o de formación médica tiene prioridad sobre la atención médica.

En las unidades médicas la demanda de atención relega a un segundo plano los procesos educativos. Situación que también se ve reflejada en las condiciones de los profesores para cumplir con su función educativa: 13\% de internos, 18\% de pasantes y 9\% de residentes consideran que sus profesores en las unidades médicas tienen las condiciones adecuadas para cumplir con sus obligaciones educativas. Lo anterior se refleja en la proporción de estudiantes que opinan que siempre recibieron atención eficaz a las dudas surgidas durante la atención de los pacientes ( $26 \%$ de internos, 36\% de pasantes y $24 \%$ de residentes). Si se pregunta por la atención que recibieron de los profesores de la Facultad de Medicina en la que están inscritos, el porcentaje disminuye a 12, 16 y 14\% respectivamente. 
Tabla 1

Número de estudiantes de medicina en unidades médicas que contestaron "siempre" al ítem correspondiente. Universidad Veracruzana, 2018-2019

\begin{tabular}{|l|c|c|c|}
\hline \multicolumn{1}{|c|}{ Ítem } & $\begin{array}{c}\text { Internos } \\
(\mathrm{n}=478)\end{array}$ & $\begin{array}{c}\text { Pasantes } \\
(\mathrm{n}=141)\end{array}$ & $\begin{array}{c}\text { Residentes } \\
(\mathrm{n}=605)\end{array}$ \\
\hline $\begin{array}{l}\text { 3. En la unidad médica en la que estuve como médico } \\
\text { en formación se priorizó el proceso de enseñanza- } \\
\text { aprendizaje sobre la demanda de atención médica. }\end{array}$ & 49 & 21 & 65 \\
\hline $\begin{array}{l}\text { 10. Mis profesores de la unidad médica tuvieron las } \\
\text { condiciones laborales adecuadas para cumplir con su } \\
\text { función educativa. }\end{array}$ & 62 & 26 & 56 \\
\hline $\begin{array}{l}\text { 1. Mis profesores en la unidad médica atendieron (de } \\
\text { manera presencial o a distancia) eficazmente las } \\
\text { dudas que tuve durante los procesos de atención } \\
\text { médica en los que participé. }\end{array}$ & 124 & 51 & 147 \\
\hline $\begin{array}{l}\text { 2. Mis profesores o tutores de la Facultad de Medicina } \\
\text { estuvieron pendientes de mi desarrollo académico en } \\
\text { la unidad médica. }\end{array}$ & 58 & 22 & 83 \\
\hline
\end{tabular}

Fuente: Elaboración propia.

La falta de condiciones para desarrollar el proceso educativo formal en las unidades médicas, la falta de tiempo para que los médicos de la unidad atiendan, supervisen y asesoren estudiantes y la poca atención de las facultades de Medicina a los estudiantes en las unidades médicas se refleja en errores diagnósticos y terapéuticos durante los procesos de educación y atención médicas. En la Tabla 2 se ve que la mayoría de estudiantes de medicina en las unidades médicas aceptan haber cometido errores médicos durante sus actividades asistenciales, ya sea por falta de supervisión o de competencias clínicas. 
Tabla 2

Número de estudiantes de medicina en unidades médicas según nivel académico que contestaron "nunca" al ítem correspondiente. Universidad Veracruzana, 2018-2019

\begin{tabular}{|l|c|c|c|}
\hline \multicolumn{1}{|c|}{ Ítem } & $\begin{array}{c}\text { Internos } \\
(\mathrm{n}=478)\end{array}$ & $\begin{array}{c}\text { Pasantes } \\
(\mathrm{n}=141)\end{array}$ & $\begin{array}{c}\text { Residentes } \\
(\mathrm{n}=605)\end{array}$ \\
\hline $\begin{array}{l}\text { Cometí errores diagnósticos o terapéuticos por falta } \\
\text { de supervisión académica durante las actividades } \\
\text { asistenciales que realicé en la unidad médica. }\end{array}$ & 148 & 66 & 148 \\
\hline $\begin{array}{l}\text { Cometí errores diagnósticos o terapéuticos por falta } \\
\text { de competencias clínicas. }\end{array}$ & 122 & 59 & 155 \\
\hline
\end{tabular}

Fuente: Elaboración propia.

Los resultados expuestos evidencian que el desarrollo del proceso educativo en las unidades médicas tiene grandes áreas de oportunidad. El aprovechamiento de estas se reflejará, indudablemente, en una mejor formación profesional de los egresados de los programas educativos de medicina general y de especialidades médicas.

\section{Acreditación de las facultades de medicina de la universidad veracruzana}

De manera cierta, la educación médica en la UV ha tenido avances y logros importantes en los años recientes. Actualmente las cinco facultades de Medicina están acreditadas por parte del Consejo Mexicano para la Acreditación de la Educación Médica, A. C. (COMAEM).

Desde la perspectiva del ESBDH es importante mencionar que la educación médica únicamente cumple con su cometido y con su responsabilidad social cuando forma médicos competentes. En este sentido, es necesario apreciar en su justo valor la acreditación, misma que siempre debe ser considerada como un medio y no como un fin. Es indudable que acreditar un programa educativo crea condiciones para una mejor formación de médicos, pero no la garantiza (Vázquez F, Ortiz MC. 2018a). Esto es, hay programas acreditados que tienen egresados con mal desempeño en exámenes nacionales de medicina y hay programas de formación médica no acreditados con egresados que tienen un buen desempeño en los mismos. En este sentido, y desde la perspectiva del ESBDH, se insiste en que el fin de los programas de educación médica es la competencia profesional de sus egresados y para ello el OBEME desarrolló un catálogo o registro de programas de educación médica en México, según el desempeño de sus egresados en las evaluaciones nacionales (Vázquez F, Ortiz MC. 2018b).

En el registro de programas de educación médica que confirman la competencia profesional de sus egresados con evaluaciones externas se observa que, de las cinco facultades de 
Medicina de la UV, únicamente la Facultad de Medicina de Minatitlán ofrece una garantía externa sólida de formar médicos competentes. Las demás facultades de Medicina no están en el Padrón de Programas de Alto Rendimiento Académico-Medicina construido por el Centro Nacional para la Evaluación de la Educación Superior (CENEVAL), por lo que quedan en el grupo de programas con poca evidencia externa de la competencia profesional de sus egresados. Lo cierto es que los egresados de todas las facultades tienen un promedio mayor a 60 en el Examen Nacional de Aspirantes a Residencias Médicas (ENARM).

Más allá de la investigación que realizan profesores e investigadores de una escuela o facultad de Medicina, de las citas que tiene su comunidad académica, de la infraestructura que tiene, de sus redes académicas y profesionales, del prestigio social del que goza, de la aceptabilidad de sus egresados, de la o las acreditaciones que tiene, y de otros indicadores similares, es importante insistir en que la función primordial de las facultades de Medicina es formar médicos competentes. En consecuencia, el criterio fundamental para juzgar la calidad de una escuela o facultad de Medicina es la garantía externa que ofrece, independiente de la que sus propios procesos de evaluación y acreditación de estudiantes brinda, a la formación profesional que da a sus egresados.

\section{Desafíos de la covid-19 a la educación médica}

El surgimiento de la pandemia de COVID-19 a principios del 2020 cambió radicalmente el contexto de la educación médica. La permanencia de los estudiantes en las unidades médicas fue seriamente cuestionada: las unidades médicas se transformaron de espacios educativos en zonas de riesgo para la salud. En este contexto, se retiró a los estudiantes de medicina de los campos clínicos, se recurrió a la educación a distancia y surgió y configuró la posibilidad real de que la formación médica se diera totalmente de manera simulada y virtual. Surgen así múltiples preguntas y temas de educación médica a considerar.

La primera pregunta que surge, desde el ESBDH, es si los estudiantes de medicina tienen o no obligaciones deontológicas, esto es, obligación moral de participar en la respuesta social a las emergencias sanitarias. ¿Cómo valorar desde el punto de vista ético el que los estudiantes de medicina, durante una epidemia, o emergencia sanitaria, se vayan a sus casas y no colaboren a enfrentar la situación? No se trata de contestar esta pregunta en este espacio; sin embargo, es importante señalar que las obligaciones deontológicas se enseñan, se aprenden y se asumen de manera gradual; no se adquieren automáticamente a la hora de la titulación. A partir de esta premisa es importante considerar el estudio de la deontología médica que, 
desafortunadamente, aparece cada vez menos en los planes de estudio de Medicina y se resalta la necesidad de elaborar protocolos de educación médica en casos de epidemias y desastres naturales que orienten sobre el quehacer de estudiantes y profesores en situaciones extraordinarias.

Es evidente que la respuesta deontológica a la pregunta planteada no debe contraponerse, de ninguna manera, a la obligación social, institucional y personal de cuidar la salud de estudiantes y trabajadores de la salud. Al margen de que toda profesión tiene sus propios riesgos y de que es parte de la preparación profesional aprender a enfrentarlos, es evidente que hay obligación social de cuidar la salud de los estudiantes de medicina. Es más, de manera independiente a las epidemias y desastres sanitarios, hay que re-pensar y enfatizar la responsabilidad que tienen las instituciones, educativas y de salud, de la salud física y mental de los estudiantes. Resulta paradójico que los propios procesos de educación médica sean un factor de riesgo para la salud de los futuros profesionales de la salud. En este sentido, el OBEME también ha documentado las jornadas mayores a 80 horas semanales de los estudiantes en las unidades médicas, la falta de tiempo para la realización de actividades físicas y recreativas, la carencia de sueño y la mala alimentación, entre otros factores contrarios a una vida saludable (OBEME, 2019).

Además del aspecto deontológico, también relacionadas con la pandemia surgen preguntas que apuntan hacia aspectos más técnicos: ¿qué formación y preparación se ofrece actualmente a los estudiantes para enfrentar situaciones de emergencia sanitaria?, ¿hasta dónde la educación médica actual forma médicos con competencias para el manejo y la gestión de epidemias, desastres y, en general, emergencias sanitarias? De manera cierta, estas y otras preguntas desafían la educación médica actual.

Por su trascendencia en la educación médica, otro aspecto a considerar en el escenario actual es el relacionado con la educación a distancia en medicina. De manera cierta, la educación a distancia y el uso de simuladores tiene ventajas indiscutibles en la formación médica para el diagnóstico y tratamiento de las enfermedades. Sin embargo, sus alcances son limitados para el trato con los enfermos. Desde la perspectiva de los derechos humanos, el médico trata enfermos, no enfermedades. Reducir la profesión médica únicamente al estudio y tratamiento de las enfermedades ya es una degradación de la dignidad humana y un menoscabo de la profesión médica. 


\section{Conclusiones y propuestas}

El OBEME está cumpliendo el objetivo para el que fue creado; ha documentado y dado a conocer la problemática de la supervisión de estudiantes de medicina en las unidades médicas y su repercusión sobre el derecho a la salud y a la educación. Las acciones necesarias para atender las áreas de oportunidad detectadas rebasan con mucho los alcances y competencias del observatorio, por lo que es deseable una mayor comunicación entre éste y las instancias responsables de la atención a las oportunidades expuestas. Al respecto, se sugiere establecer el consejo consultivo del OBEME con atribuciones para realizar la planeación-evaluación de las actividades del observatorio y de los programas de atención a los problemas diagnosticados.

\section{II}

Únicamente una de las cinco facultades de Medicina de la UV valida por una instancia externa las competencias profesionales de sus egresados. Por lo que, más allá de la acreditación, es importante que todas las facultades realicen las gestiones para su incorporación al Padrón de Programas de Alto Rendimiento Académico-Medicina. A la fecha, en México, esta es la manera más confiable y válida para evaluar y certificar por una instancia externa las competencias y la calidad de los egresados de los programas de formación médica. Adicionalmente y en este mismo sentido, se sugiere instituir un reconocimiento anual para reconocer a la Facultad de Medicina de la UV que tenga los mejores resultados.

\section{III}

La emergencia de la COVID-19: 1) ha dibujado de manera clara la necesidad de incluir lo relacionado con la gestión de epidemias y desastres en el plan de estudios, 2) hace ver la necesidad de definir, desde la deontología, la participación de los estudiantes en las emergencias sanitarias y elaborar protocolos de acción para la educación médica en situaciones extraordinarias y 3) ha evidenciado la importancia y la responsabilidad institucional de cuidar, con o sin pandemia, la salud de los estudiantes de medicina.

\section{Referencias}

Boelen Ch, Woollard R. (2011). Consenso Global sobre la Responsabilidad Social de las Facultades de Medicina. Educación Médica, 14(1), 7-14. 
Comisión Nacional de Derechos Humanos (2009). Recomendación General No. 15. Sobre el derecho a la protección de la salud. Recuperada: 13 enero 2017. De: http://www.cndh.org.mx/sites/all/doc/Recomendaciones/generales/RecGral_015.pdf

OBEME. (2019). Educación Médica y Derechos Humanos. Evaluación del respeto a los derechos humanos de estudiantes de medicina de la Universidad Veracruzana, en las unidades de atención médica (2017-2018). Xalapa, Ver.: Instituto de Salud Pública. Universidad Veracruzana. Recuperado de https://www.uv.mx/obeme/files/2019/08/InformeEducacion-Medica-Derechos-Humanos.pdf

Vázquez F. (2017). Reflexiones que justifican la constitución del Observatorio de Educación Médica y Derechos Humanos. UVserva, No 3, 111-113. Recuperado de https://uvserva.uv.mx/index.php/Uvserva/article/view/2541/4423

Vázquez F, Ortiz MC. (2018a). Acreditación y resultados de la educación médica en México. Educación Médica, 19(6), 333-338. Doi: https://doi.org/10.1016/j.edumed.2017.04.016

Vázquez F, Ortiz MC. (2018b). Registro de programas de educación médica que confirman la competencia profesional de sus egresados con evaluaciones externas, 2017. UVserva, No 5, 35-42. Recuperado de https://uvserva.uv.mx/index.php/Uvserva/article/view/2571/4453

Yamin EA, Frisancho A. (2015). Enfoques basados en los derechos humanos para la salud en América Latina. Medicc Review, 17, Suplemento., S5-7. 